\title{
DESIGUALDADES DE RENDA: A ESCOLARIDADE EM QUESTÃO*
}

\author{
Marcelo Gomes Ribeiro ${ }^{1}$
}

\begin{abstract}
RESUMO: O trabalho objetiva discutir o efeito da escolaridade sobre as desigualdades de renda em duas metrópoles brasileiras, Rio de Janeiro e São Paulo, contrastando dois modelos analíticos: o modelo referente à teoria do capital humano e o modelo overeducation, required education e undereducation (ORU). Esse contraste procura apresentar evidências empíricas de que a escolaridade exigida pela ocupação é mais relevante do que a escolaridade do indivíduo. Porém, tanto numa situação como noutra, o efeito da escolaridade sofre alteração a depender da conjuntura do mercado de trabalho: numa situação de elevada taxa de desemprego, o efeito da escolaridade é mais elevado; o contrário se evidencia quando a taxa de desemprego é mais reduzida. Foram utilizados os dados da Pesquisa Nacional por Amostra de Domicílios, do Instituto Brasileiro de Geografia e Estatística, de 2002, 2007 e 2013.
\end{abstract}

Palavras-chave: Escolaridade dos indivíduos. Escolaridade requerida. Sobre-educação. Subeducação. Mercado de trabalho.

\section{INCOME INEQUALITY: THE EDUCATION IN QUESTION}

ABSTRACT: The work discusses the effect of education on income inequality in two Brazilian cities, Rio de Janeiro and Sao Paulo, contrasting analytical models: the model related to the theory of human capital and the overeducation, required education and undereducation (ORU) model. This contrast seeks to present empirical evidence that the education required by occupation is more relevant than individuals' level of education. However, in both situations the effect of education changes depending on the labor market condition: in a high unemployment one, the effect of education is higher; the opposite is evident when the unemployment rate is lower. Data from the National Household Sampling Survey of the Brazilian Institute of Geography and Statistics of 2002, 2007 and 2013 were used

Keywords: Individuals' education. Required education. Overeducation. Undereducation. Labor market.

\footnotetext{
*Artigo resultado do projeto de pesquisa "Mercado de Trabalho, Segmentaçáo Socioespacial e Desigualdades nas Regiôes Metropolitanas Brasileiras nas Últimas Décadas" financiado pelo CNPq. ${ }^{1}$ Universidade Federal do Rio de Janeiro, Instituto de Pesquisa e Planejamento Urbano e Regional - Rio de Janeiro (RJ), Brasil. E-mail: marceloribeiro@ippur.ufrj.br
}

DOI: 10.1590/ES0101-73302016154254 


\section{INÉGALITÉ DES REVENUS : L'ÉDUCATION EN CAUSE}

RÉSUMÉ: Ce travail traite de l'effet del'éducation sur l'inégalité des revenus dans deux villes brésiliennes, Rio de Janeiro et Sáo Paulo, en confrontant deux modèles d'analyse: le modèle lié à la théorie du capital humain et le modèle ORU (suréducation, formation requise et la sous-scolarisation). Cette confrontation tente de montrer empiriquement que l'éducation requis par la profession est plus pertinente que le niveau d'éducation de l'individu. Cependant, et quelle que soit le modèle, l'effet de l'éducation change en fonction du marché du travail: dans une situation de chômage élevé, l'effet de l'éducation est plus élevé; on observe l'inverse lorsque le taux de chômage est plus faible. Les données de l'Enquête nationale par échantillon de domiciles par l'Institut Brésilien de Géographie et Statistique (IBGE) de 2002, 2007 et 2013.

Mots-clés: Education des individus. Formation requise. Suréducation. Uneducation. Marché du travail.

\section{Introdução}

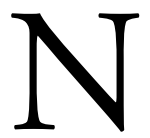

o debate público e acadêmico acerca da explicação das desigualdades de renda observadas entre os indivíduos no mercado de trabalho, tornou-se recorrente o argumento de que essas distinçóes decorrem da diferença de escolaridade observadas entre eles. Porém, há de se questionar qual é a escolaridade que importa para a explicação das diferenças de obtençáo de renda entre os indivíduos no mercado de trabalho: seria a escolaridade dos indivíduos ou a escolaridade requerida pelas ocupaçôes? Essa pergunta torna-se cada vez mais importante quando as sociedades apresentam níveis mais elevados de escolarizaçấo, como tem ocorrido no Brasil nos últimos anos. Por isso, temos como hipótese que a escolaridade exigida pela ocupação é mais importante como explicação das diferenças de renda entre os indivíduos no mercado de trabalho do que a escolaridade dos indivíduos.

O objetivo deste artigo foi confrontar dois modelos analíticos que utilizam a escolaridade para explicar os diferenciais de renda obtidos pelos indivíduos no mercado de trabalho. O primeiro modelo analítico decorre da teoria do capital humano, que se tornou importante por utilizar a escolaridade dos indivíduos para a explicação dos diferenciais de renda. $\mathrm{O}$ segundo modelo analítico considera a escolaridade requerida pelas ocupaçóes. $\mathrm{Na}$ medida em que se observa o requerimento educacional de cada ocupação, percebe-se a existência de situaçóes em que há pessoas com mais escolaridade do que a exigida pelas ocupaçóes (situação de overeducation) e situaçôes nas quais há pessoas com menor escolaridade do que a 
requerida pelas ocupações (situaçôes de undereducation). Nesse último modelo, portanto, sáo consideradas a escolaridade requerida, a situação de overeducation e a situaçáo de undereducation.

O capítulo está organizado em cinco seçôes, incluída esta introdução. $\mathrm{Na}$ segunda seção, procura-se apresentar os aspectos teóricos e analíticos da relação entre escolaridade e desigualdades de renda, focando nos mecanismos explicativos dessa relação. $\mathrm{Na}$ terceira seção, buscamos realizar a caracterização do mercado de trabalho das regióes metropolitanas de São Paulo e do Rio de Janeiro, para os anos em que a análise empírica será empreendida, referente ao comportamento da taxa de desemprego, da renda e da escolaridade da populaçáo ocupada. Na quarta seção, realizam-se os testes de hipóteses, procurando apresentar explicação para os resultados encontrados. Por fim, a última seção sintetiza os principais resultados na perspectiva das consideraçóes finais.

\section{Considerações analíticas dos retornos monetários da escolaridade}

Tornou-se consagrada na literatura especializada a utilização da teoria do capital humano para explicação dos diferenciais de rendimentos obtidos pelos indivíduos no mercado de trabalho. Essa teoria foi apresentada inicialmente por Jacob Mincer (1958) e popularizada por Theodore W. Schultz (1961) e Gary S. Becker (1964). Porém, o avanço dessa perspectiva teórica se deu a partir da modelagem econométrica formulada por Jacob Mincer em seu Schooling, Experience, and Earnings, em $1974^{1}$.

Tanto no trabalho de Mincer (1974) quanto em outros que utilizaram esse modelo analítico para mensurar os retornos monetários dos indivíduos por decorrência do seu investimento em capital humano, os retornos da escolaridade apresentam resultados positivos, atestando que cada ano a mais de escolaridade aumenta o nível de remuneraçáo dos indivíduos. A aplicação do modelo minceriano foi realizada em diversos países e os resultados se mostraram muito semelhantes, apresentando retornos monetários que variam entre 5 e $15 \%$ resultantes da elevação em cada ano de escolaridade dos indivíduos (GROSSBARD, 2006). A interpretação dada aos retornos educacionais sobre os rendimentos é feita no sentido de atribuir aos indivíduos mais escolarizados maior produtividade de trabalho, o que justificaria o fato daqueles com maior nível de instrução auferirem maior remuneração do que outros com menor nível de instruçâo. Essa maior produtividade dos indivíduos de maior escolaridade é decorrente, nesse sentido, da formação escolar obtida ao longo do processo educacional, traduzida em maiores retornos monetários, como prêmio pelo esforço e tempo dedicado à educação.

O aumento generalizado do nível de instrução da população dos países mais desenvolvidos, levou, no final da década de 1970, ao questionamento da perspectiva teórica do capital humano para a explicação dos diferenciais de 
rendimento entre os indivíduos no mercado de trabalho. Isso se deu à proporção que essa perspectiva, ao se apoiar em variáveis referentes apenas à oferta de trabalho - correspondente às características dos indivíduos —, desconsidera os aspectos relativos à demanda por trabalho.

$\mathrm{Na}$ verdade, verificou-se que por decorrência do aumento do nível de instrução da população desses países havia muitos trabalhadores com nível mais elevado de instrução do que o exigido pela ocupação em que trabalhavam, tornando incompatível, em muitos casos, a escolaridade dos trabalhadores e a escolaridade requerida por suas respectivas ocupaçóes. Isso ocorria porque, na medida em que o mercado de trabalho passava a ofertar trabalhadores mais instruídos, seria satisfatório demandar mão de obra com maior nível de instrução, mesmo que incompatível com a escolaridade exigida pela ocupação. Nesse sentido, buscou-se considerar que mais importante do que analisar a escolaridade adquirida pelos trabalhadores - característica da oferta de trabalho - seria analisar a escolaridade requerida pela ocupação, isto é, o aspecto da demanda de trabalho.

Duncan e Hoffman (1981) foram os primeiros autores a elaborarem um modelo analítico que possibilitou verificar o efeito da educação para aferir os diferenciais de rendimento entre os indivíduos nas condiçóes em que o nível de instrução eleva-se de modo generalizado, provocando, em muitas circunstâncias, a ocorrência de overeducation (sobre-educação), que corresponde à situação em que os trabalhadores possuem escolaridade acima da exigida pela ocupação. Além disso, no modelo proposto consideraram também a existência de undereducation (subeducação) - escolaridade do indivíduo inferior à exigida pela ocupação - e de educação requerida pela ocupação ${ }^{2}$.

$\mathrm{Na}$ análise realizada para os Estados Unidos da América, a partir de uma pesquisa longitudinal decorrente do Panel Study of Income Dynamics e com uma amostra representativa nacional de 5 mil domicílios, verificou-se que em torno de $40 \%$ da força de trabalho americana possuía maior nível de instrução em relação à exigência de escolaridade de suas ocupaçóes. Para tanto, propuseram um modelo que substituísse no modelo minceriano os anos de escolaridade adquiridos pelos indivíduos por anos de escolaridade que a ocupação requeria, assim como os anos de escolaridade para situações de sobre-educação e subeducação. Esse modelo ficou conhecido como over, required and under-education (ORU).

Hartog (2000) constata que várias pesquisas foram realizadas posteriormente utilizando o modelo analítico da ORU, trazendo desdobramentos importantes para análise dos determinantes dos diferenciais de rendimento entre os indivíduos. Porém, apresenta que há ainda muitos desafios a serem enfrentados para o estabelecimento desse modelo como explicativo dos diferenciais de rendimento no mercado de trabalho. Mesmo assim, Hartog (2000) já o considera como muito relevante por levar em conta o lado da demanda do mercado de trabalho, o que não é feito pela teoria do capital humano. 
O principal desafio enfrentado pelas pesquisas que utilizaram o modelo da ORU decorre da definição da escolaridade requerida e, por sua vez, do que é compreendido por sobre-educação e subeducação. $\mathrm{Na}$ literatura utilizada para realizar a resenha dos desdobramentos da ORU, Hartog (2000) afirma que encontrou três tipos diferentes para definir suas variáveis-chave: job analysis (JA), worker self-assessment (WA) e realized matches (RM). O primeiro método de definição da ORU decorre do uso de dicionários de ocupaçóes feitos por especialistas do trabalho. O segundo método é obtido por meio de informaçóes do próprio trabalhador sobre o que ele considera como escolaridade requerida para a ocupação que realiza. O terceiro decorre do uso indireto da escolaridade requerida pela ocupação, por meio da combinação de outras variáveis, tais como a média (ou a moda) da escolaridade das ocupaçóes. Ao salientar as vantagens e desvantagens de cada um desses métodos, considera-se que o mais robusto diz respeito ao método job analysis (JA).

A questáo implicada em cada um desses métodos decorre de como se pode definir a escolaridade requerida de cada uma das ocupaçóes. Essa é uma questão importante porque nem sempre as ocupaçôes apresentam exigência de escolaridade bem definida, o que pode implicar em sobrevalorização ou subvalorização do nível de instrução atribuído. Além disso, observa-se que há, em muitos casos, variabilidade grande entre os anos de instrução dos indivíduos que estão em ocupaçáo correspondente. Por isso, a definição da escolaridade requerida é o primeiro desafio a ser enfrentado quando se procura utilizar o modelo analítico da ORU. E é por decorrência dessa definição que se pode, inclusive, definir as demais variáveis do modelo: sobre-educação e subeducação.

Hartog (2000) também menciona outra maneira de definir as variáveis da ORU, decorrente dos estudos realizados por Verdugo e Verdugo (1989). Diferente do que foi apresentado anteriormente, esses autores, ao invés de utilizarem a escolaridade requerida pela ocupação, consideram a escolaridade adquirida pelos trabalhadores de cada ocupação para analisar os diferenciais de ganhos. Além disso, definem sobre e subeducação de acordo com a diferença entre a escolaridade adquirida e a média, somada (ou subtraída) de um desvio padrão da escolaridade. Porém, nos estudos empíricos, os autores dão tratamento às variáveis de sobre e subeducação como variáveis dummy (categóricas), ao contrário de as utilizarem como anos de estudo (variáveis discretas). Hartog (2000), por considerar menos atrativo esse método, se atém mais nas pesquisas que utilizam a ORU como mencionado anteriormente.

De modo geral, Hartog (2000) verifica que as pesquisas que se valem da ORU apresentam as seguintes conclusóes empíricas:

- os retornos da escolaridade requerida são maiores do que os retornos da educação adquirida pelos trabalhadores (ao se comparar com o modelo Minceriano); 
- os retornos da sobre-educação são positivos, mas menores do que os da educação requerida. Tipicamente, os retornos da sobre-educação equivalem ou à metade, ou a dois terços dos retornos da educação requerida;

- os retornos da subeducação são negativos. A penalidade pela subeducação é sempre menor do que a dos retornos pela educação requerida e também menor do que os retornos pela sobre-educação.

Essas constatações empíricas podem ser interpretadas, segundo Hartog (2000), do seguinte modo:

1. Em uma dada ocupação, com um dado nível de escolaridade requerida, os ganhos para trabalhadores subeducados são menores e os ganhos para trabalhadores sobre-educados são maiores, quando comparados aos trabalhadores que trazem apenas a escolaridade requerida pelo trabalho.

2. Trabalhadores com dada escolaridade que conseguem uma ocupação que requer maior escolaridade do que eles possuem têm maiores ganhos do que aqueles com o mesmo nível de escolaridade num trabalho compatível com sua escolaridade (efeito positivo da sobre-educação). Entretanto, conseguem menores ganhos do que os trabalhadores que têm a escolaridade requerida pelo trabalho que realizam (sobre-educaçâo tem menores retornos do que a escolaridade requerida).

Diaz e Machado (2008), ao se basearem em McGuinness (2006), procuram interpretar esses resultados de acordo com o modelo de competiçáo por emprego. De acordo com esses autores:

\begin{abstract}
Segundo McGuinness (2006), o Modelo de Competição por Emprego fornece uma clara explicação teórica para o fenômeno da sobre-educação na medida em que se fundamenta na premissa de que os indivíduos competem pelas oportunidades de emprego baseando-se nos seus custos relativos de treinamento, e não na remuneraçáo que estariam dispostos a aceitar dado seu nível de escolaridade. Deste modo, quanto maior for o número de indivíduos educados na população maior será a necessidade individual de investir em educação como forma de preservar sua posição. (DIAS; MACHADO, 2008, p. 422)
\end{abstract}

Essa linha de argumentação do modelo de competição por emprego corresponde ao que também é denominado de teoria da fila, formulada por Thurow (1972). Apesar de Thurow não ter desenvolvido um modelo analítico capaz de incorporar a dimensão da demanda do mercado de trabalho, suas formulaçóes contribuem para a interpretaçấo da ORU em direção contrária à interpretação adotada pelo capital humano. 
$\mathrm{Na}$ medida em que ocorre aumento generalizado do nível de instrução da população e, por conseguinte, aumento da sobre-educação e redução da subeducaçáo, como observado por Hartog (2000) na comparaçáo entre alguns países europeus, o modelo de competição por emprego (ou da fila) possibilita uma interpretação mais adequada dos resultados encontrados no modelo ORU. Isso porque, se os empresários utilizam a educação para sinalizar treinabilidade, quanto mais elevado o nível de instrução da população, maior será a probabilidade de selecionarem os mais escolarizados, dada a grande oferta de mão de obra com alto nível de instrução.

No Brasil, as pesquisas que utilizam o modelo da ORU são ainda poucas e recentes, porque o aumento de pessoas com elevado nível de instrução ocorreu tardiamente no país, se comparado com os países desenvolvidos. Mesmo assim, destacam-se importantes análises já realizadas que se utilizaram de métodos diferentes para definição da educação requerida, da sobre-educação e da subeducação. O trabalho pioneiro que considerou a situação de sobre-educação e de subeducação no Brasil é de Santos (2002), mas também podemos apontar os trabalhos de Machado, Oliveira e Carvalho (2003), de Diaz e Machado (2008), de Esteves (2009) e de Vianna e Oliveira (2010).

Como podemos observar, a formulação de um novo modelo analítico para a compreensão dos efeitos explicativos dos diferenciais de rendimento do trabalho avançou nas últimas décadas ao estabelecer a discussão pelo lado da demanda do mercado de trabalho. Assim, os resultados desse novo modelo analítico se tornaram importantes como contraposição à perspectiva da teoria do capital humano, que considera apenas o lado da oferta do mercado de trabalho. Porém, como vimos, a análise dos resultados ainda é carente de interpretação teórica mais robusta, apesar da perspectiva de Thurow contribuir nessa direção. Nesse sentido, torna-se importante continuar avançando nas perspectivas que colaboram para tal interpretação.

\section{Conjunturas do mercado de trabalho}

Para realizar a análise empírica e interpretativa da relação entre escolaridade e renda, é necessário considerar as situaçôes de conjuntura do mercado de trabalho, principalmente no que se refere aos aspectos do desemprego, da renda e da escolaridade. A primeira década do século XXI apresentou mudanças importantes no mercado de trabalho brasileiro, mas especialmente nos principais centros urbanos do país, como são os casos das regiốes metropolitanas de São Paulo e do Rio de Janeiro. Essas mudanças, de modo geral, decorreram da maior formalizaçáo do trabalho, da redução da taxa de desemprego e da reduçáo das desigualdades de renda.

Podemos observar no Gráfico 1 que a taxa de desemprego nessas duas metrópoles tinha patamares muito elevados em 2002, sendo que na região metro- 


\section{Gráfico 1}

Taxa de desemprego de períodos selecionados nas regiôes metropolitanas de São Paulo e do Rio de Janeiro.

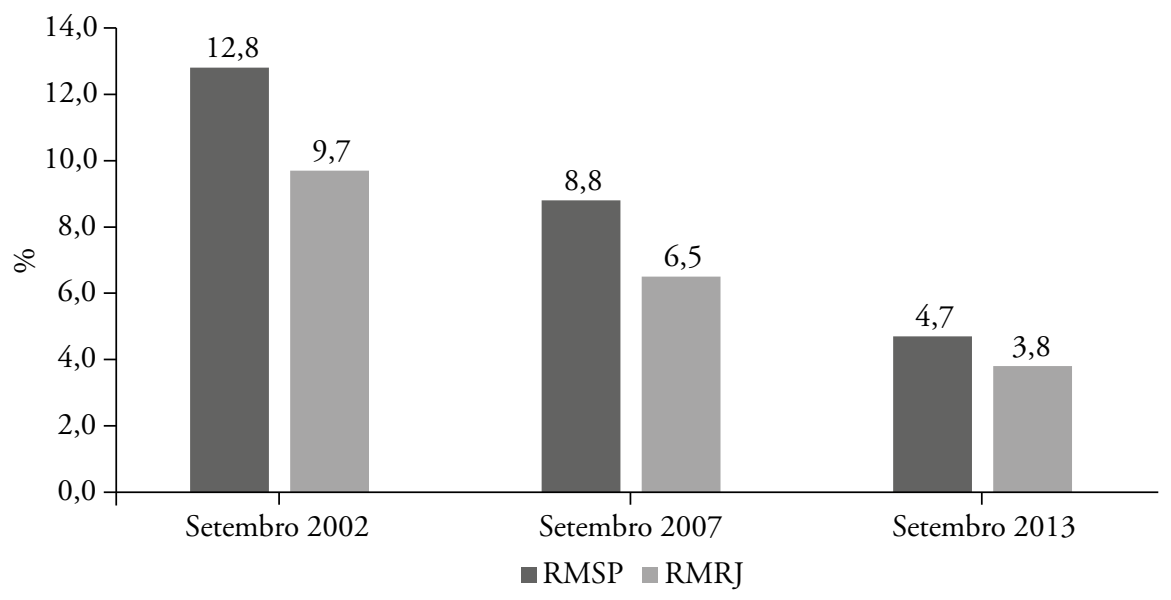

RMSP: regiäo metropolitana de São Paulo; RMRJ: regiäo metropolitana do Rio de Janeiro. Fonte: IBGE (2002a; 2007a; 2013a).

politana de São Paulo ela atingiu patamares de dois dígitos, o mesmo ocorrendo similarmente na região metropolitana do Rio de Janeiro. Ao longo desse período, houve sistemática redução da taxa de desemprego, atingindo níveis de 4,7\% na regiāo metropolitana de São Paulo e de 3,8\% na região metropolitana do Rio de Janeiro, em setembro de 2013.

O momento em que a taxa de desemprego encontrava-se elevada pode ser caracterizado por uma conjuntura econômica contracionista, na medida em que nessas situaçóes o crescimento econômico tende a ser mais reduzido. Ao contrário, quando a taxa de desemprego apresenta níveis reduzidos, a conjuntura econômica pode ser caracterizada como expansionista. O que se espera é que em conjunturas contracionistas a escolaridade passe a ser um importante ativo para os indivíduos se inserirem no mercado de trabalho e, mais do que isso, para obtençáo de maior nível de remuneração. Por outro lado, quando o mercado de trabalho está em expansão, espera-se que haja desvalorizaçáo da escolaridade e, por conseguinte, ela deixa de ser um atributo relevante para explicaçáo dos diferenciais de renda entre os indivíduos. De qualquer modo, para análise mais precisa dos retornos da escolaridade sobre os diferenciais de renda, é necessário que seja considerado também o comportamento da renda ao longo do período que está sendo analisado e também as mudanças ocorridas em relação ao nível educacional da população.

De maneira geral, o rendimento médio do trabalho principal sofreu aumento real no período de 2002 a 2013, como podemos confirmar no Gráfico 2, tanto para a regiấo metropolitana do Rio de Janeiro quanto para a região metro- 


\section{Gráfico 2}

Rendimento médio do trabalho principal de pessoas de15 anos e mais de idade nas regióes metropolitanas de São Paulo e do Rio de Janeiro.

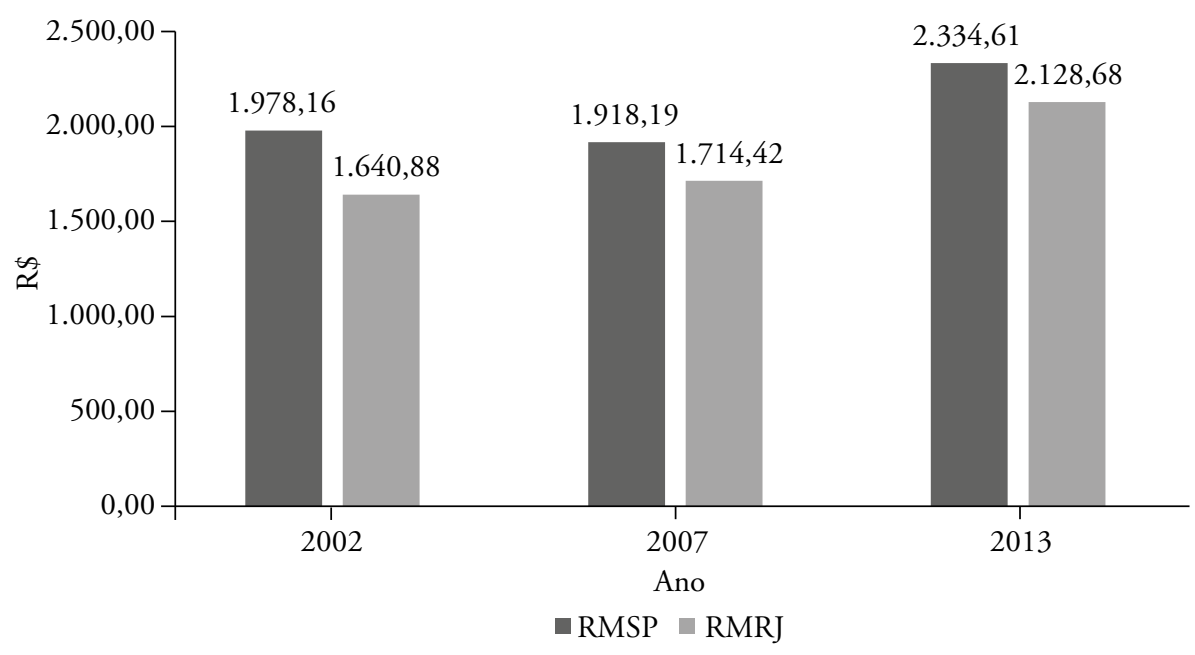

RMSP: região metropolitana de São Paulo; RMRJ: região metropolitana do Rio de Janeiro. Fonte: IBGE (2002b; 2007b; 2013b).

Nota: Valores corrigido pelo Índice Nacional de Preços ao Consumidor de setembro de 2014.

politana de São Paulo. Nessa última, entretanto, ocorreu pequena redução entre 2002 e 2007, mas com posterior aumento em 2013, atingindo patamar superior ao rendimento médio de 2002.

A metrópole fluminense teve um aumento real no rendimento médio do trabalho principal de 30\% entre setembro de 2002 e setembro de 2013; já na metrópole paulista o aumento real foi de $18 \%$. Mesmo que no Rio de Janeiro o crescimento do nível de renda tenha sido superior, o rendimento médio do trabalho principal da regiáo metropolitana de São Paulo continuou superior na comparaçáo com o rendimento médio da regiáo metropolitana do Rio de Janeiro.

Quando se observa a distribuição relativa da população ocupada segundo os anos de escolaridade, tanto na regiáo metropolitana do Rio de Janeiro quanto na regiáo metropolitana de São Paulo, percebe-se que tem havido, ao longo do período analisado, aumento da proporção de pessoas que possuem níveis mais elevados de escolaridade e, por conseguinte, redução da proporção de pessoas com níveis mais inferiores. Como podemos ver na Tabela 1 , ao considerar aqueles anos de escolaridade que demarcam mudanças de fase escolar ${ }^{3}$, percebemos que há redução da proporção de pessoas com 8 anos de escolaridade, aumento da proporção de pessoas com 11 anos de escolaridade e também aumento da proporção de pessoas com 15 anos de escolaridade. Essa situaçáo colaborou para que ocorresse 


\section{Tabela 1}

Anos de escolaridade e média de escolaridade de pessoas ocupadas de 15 anos e mais de idade, de anos selecionados, nas regióes metropolitanas de São Paulo e Rio de Janeiro.

\begin{tabular}{|c|c|c|c|c|c|c|}
\hline \multirow[t]{2}{*}{ Anos de escolaridade } & \multicolumn{3}{|c|}{$\begin{array}{l}\text { Regiáo metropolitana } \\
\text { do Rio de Janeiro }\end{array}$} & \multicolumn{3}{|c|}{$\begin{array}{c}\text { Regiáo metropolitana } \\
\text { de São Paulo }\end{array}$} \\
\hline & 2002 & 2007 & 2013 & 2002 & 2007 & 2013 \\
\hline 0 & 3,6 & 2,9 & 3,3 & 4,7 & 3,2 & 4,0 \\
\hline 1 & 0,8 & 0,7 & 0,6 & 1,2 & 1,1 & 0,5 \\
\hline 2 & 2,5 & 1,9 & 0,8 & 2,0 & 1,4 & 1,0 \\
\hline 3 & 5,2 & 3,0 & 1,9 & 3,4 & 2,1 & 1,1 \\
\hline 4 & 8,1 & 6,8 & 5,0 & 11,0 & 8,1 & 5,2 \\
\hline 5 & 8,2 & 5,2 & 4,2 & 4,9 & 4,1 & 2,8 \\
\hline 6 & 3,9 & 3,5 & 2,9 & 4,4 & 2,6 & 2,3 \\
\hline 7 & 4,4 & 3,7 & 3,2 & 4,6 & 3,3 & 2,3 \\
\hline 8 & 13,7 & 13,4 & 10,3 & 12,0 & 11,3 & 11,2 \\
\hline 9 & 2,5 & 2,6 & 2,6 & 3,3 & 2,9 & 2,4 \\
\hline 10 & 3,9 & 3,6 & 3,7 & 4,3 & 4,0 & 3,2 \\
\hline 11 & 25,2 & 30,3 & 32,6 & 25,1 & 33,6 & 35,1 \\
\hline 12 & 1,8 & 2,5 & 4,7 & 2,5 & 2,8 & 3,8 \\
\hline 13 & 1,6 & 2,1 & 2,0 & 1,9 & 2,3 & 2,7 \\
\hline 14 & 1,6 & 2,3 & 2,8 & 1,9 & 2,5 & 2,8 \\
\hline 15 & 11,1 & 13,4 & 17,2 & 11,5 & 13,5 & 17,9 \\
\hline 16 & 1,8 & 2,2 & 2,2 & 1,3 & 1,3 & 1,6 \\
\hline Total & 100,0 & 100,0 & 100,0 & 100,0 & 100,0 & 100,0 \\
\hline Média & 8,6 & 9,4 & 10,0 & 8,6 & 9,5 & 10,1 \\
\hline
\end{tabular}

Fonte: Pesquisa Nacional por Amostra de Domicílios (IBGE, 2002b, 2007b, 2013b).

elevação da média de escolaridade nas duas metrópoles analisadas, passando de uma média de 8,6, em 2002 para em torno de 10 anos de escolaridade, em 2013.

Porém, no contexto do mercado de trabalho, mais do que examinar propriamente a escolaridade dos indivíduos, é importante analisar o comportamento da correspondência dessa escolaridade com a exigência de escolaridade feita pelas ocupaçóes, porque a partir disso poderemos verificar se está havendo correspondência dos atributos educacionais oferecidos pela população com aquilo que o mercado de trabalho tem requerido. Como podemos observar na Tabela 2, nem todas as pessoas que estáo ocupadas no mercado de trabalho apresentam escolaridade compatível com a exigência da ocupação, pois parte da população 


\section{Tabela 2}

Incidência de adequação de escolaridade, sobre-educação e subeducação de pessoas ocupadas de 15 e mais anos de idade, de anos selecionados, nas regióes metropolitanas do Rio de Janeiro e de São Paulo.

\begin{tabular}{l|c|c|c|c|c|c}
\hline \multirow{2}{*}{ Situaçáo } & \multicolumn{3}{|c|}{$\begin{array}{c}\text { Regiáo metropolitana } \\
\text { do Rio de Janeiro }\end{array}$} & \multicolumn{3}{c}{$\begin{array}{c}\text { Regiáo metropolitana } \\
\text { de Sáo Paulo }\end{array}$} \\
\cline { 2 - 7 } & $\mathbf{2 0 0 2}$ & $\mathbf{2 0 0 7}$ & $\mathbf{2 0 1 3}$ & $\mathbf{2 0 0 2}$ & $\mathbf{2 0 0 7}$ & $\mathbf{2 0 1 3}$ \\
\hline Escolaridade adequada & 36,7 & 38,5 & 40,9 & 36,2 & 39,2 & 41,8 \\
\hline Sobre-educação & 23,6 & 29,5 & 34,6 & 23,3 & 30,4 & 34,0 \\
\hline Subeducaçáo & 39,7 & 32,0 & 24,5 & 40,5 & 30,4 & 24,2 \\
\hline Total & 100,0 & 100,0 & 100,0 & 100,0 & 100,0 & 100,0 \\
\hline
\end{tabular}

Fonte: Pesquisa Nacional por Amostra de Domicílios (IBGE, 2002b, 2007b, 2013b).

encontra-se em situação de sobre-educação enquanto outra parte está em situação de subeducação. Isso significa que há situaçôes de pessoas com mais escolaridade do que a exigência da ocupação, bem como aponta a existência de situaçóes de pessoas com menos escolaridade do que a exigida pela ocupação.

Além disso, podemos perceber que há considerável aumento de pessoas em situação de sobre-educação em ambas as metrópoles, passando de uma proporçáo de 23\% em 2002 para 34\% em 2013, aproximando-se de níveis observados nos países desenvolvidos, que registram essa proporção em torno de $40 \%$. Ao mesmo tempo, tem havido redução da proporção de pessoas em situação de subeducação, também nas duas metrópoles, passando de cerca de 40\% em 2002 para $24 \%$ em 2013. Do mesmo modo, tem ocorrido aumento da proporçáo de pessoas que se encontram em situação de adequação da escolaridade em relação à exigência da ocupação, atingindo, ao final do período analisado, em torno de $40 \%$ da população ocupada.

Mudanças do nível de instrução de uma população podem colaborar para alteraçóes no grau de desigualdade de rendimento entre seus indivíduos? Tal pergunta tem sido tema de discussáo cada vez maior no país desde a década anterior, quando aumentou a taxa de desemprego e, para a obtenção de trabalho, passou-se a exigir cada vez mais maior escolaridade. Mas essa discussão também tem se mantido quando no mercado de trabalho passou-se a observar reduçáo do desemprego, acompanhado do aumento da participação da populaçáo economicamente ativa e da reduçáo das desigualdades de rendimento oriundo do trabalho.

Como já fora assinalado, a interpretação dos mecanismos que explicam a relação entre educação e diferencial de rendimentos obtidos no mercado de trabalho se organiza sob diferentes e, por vezes, antagônicas perspectivas teóricas. Ao recorrer àquelas que ajudam a interpretar a relação entre educação e retornos 
monetários, é preciso considerar, todavia, que estamos diante de um fato social que não pode ser negligenciado: apesar de haver reclamação de que faltam pessoas escolarizadas no mercado de trabalho, tem-se percebido uma elevação sistemática do nível de instruçấo da população brasileira, principalmente quando analisada nos espaços metropolitanos do país.

\section{Efeitos da escolaridade sobre os diferenciais de renda}

Para análise do efeito da educação sobre os diferenciais de renda obtidos pelos indivíduos no mercado de trabalho, o procedimento operacional para realização dos testes de hipóteses neste trabalho consiste na efetuação de análise de dados secundários decorrentes de pesquisas domiciliares do tipo survey, realizadas pelo Instituto Brasileiro de Geografia e Estatística (IBGE), que compõem a Pesquisa Nacional por Amostra de Domicílios (PNAD). Para tanto, foi confrontado o efeito da escolaridade de dois modelos analíticos (Mincer e ORU, esse último segundo a job analysis), que correspondem à análise de regressão linear múltipla, baseada no método de mínimos quadrados ordinários (MQO). A regressão linear múltipla nos possibilita testar o efeito conjugado de diversas variáveis, denominadas independentes ou explicativas, sobre a variável dependente ou variável a ser explicada. A consideração necessária é que a variável dependente seja contínua, o que corresponde à característica do logaritmo do rendimento do trabalho principal - a variável dependente de ambos os modelos analíticos.

Esse procedimento estatístico foi aplicado para os anos de 2002, 2007 e 2013, de modo a compararmos o efeito das variáveis independentes em momentos em que o mercado de trabalho encontrava-se em contração - caracterizada pela elevada taxa de desemprego - e em momentos em que o mercado de trabalho apresentava característica expansionista - quando a taxa de desemprego é reduzida. Todas as variáveis utilizadas são decorrentes da PNAD ${ }^{4}$ : a variável dependente testada nos dois modelos analíticos foi o logaritmo do rendimento do trabalho principal por hora; a variável dependente do modelo de Mincer corresponde aos anos de escolaridade dos indivíduos; a variável dependente do modelo ORU equivale aos anos de escolaridade requerida pela ocupação, situação de sobre-educação e situação de subeducação. Em todos os modelos foram acrescentadas variáveis de controle, que se referem a variáveis de discriminação no mercado de trabalho (sexo, cor ou raça, posição social e localização residencial no território metropolitano, além da experiência de trabalho e da experiência de trabalho ao quadrado).

No Gráfico 3, referente às regiôes metropolitanas do Rio de Janeiro e de São Paulo, apresentamos os resultados do efeito da escolaridade sobre os diferenciais de rendimento para os dois modelos analíticos, de pessoas ocupadas 


\section{Gráfico 3}

Efeito da escolaridade dos indivíduos, da escolaridade requerida pela ocupaçáo, da sobre-educação e da subeducação sobre os diferenciais de rendimento das pessoas ocupadas na semana de referência no mercado de trabalho, de 15 anos e mais de idade, para as regióes metropolitanas do Rio de Janeiro e de São Paulo - 2002, 2007 e 2013.

Regiāo metropolitana do Rio de Janeiro
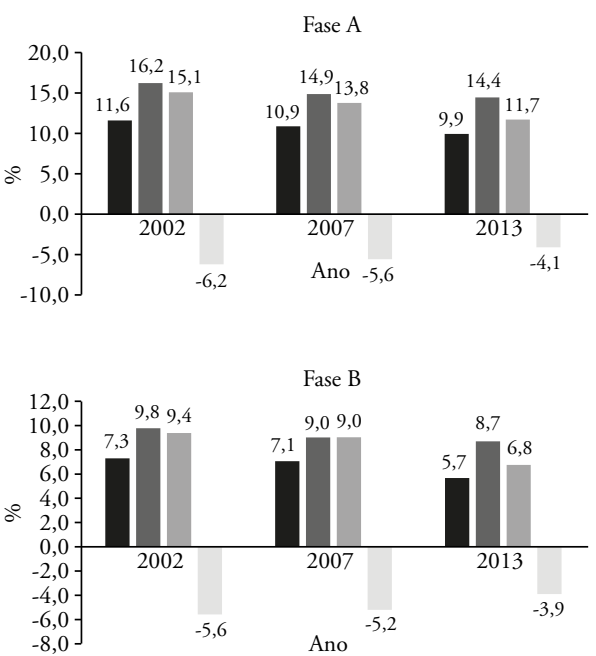

Região metropolitana de São Paulo
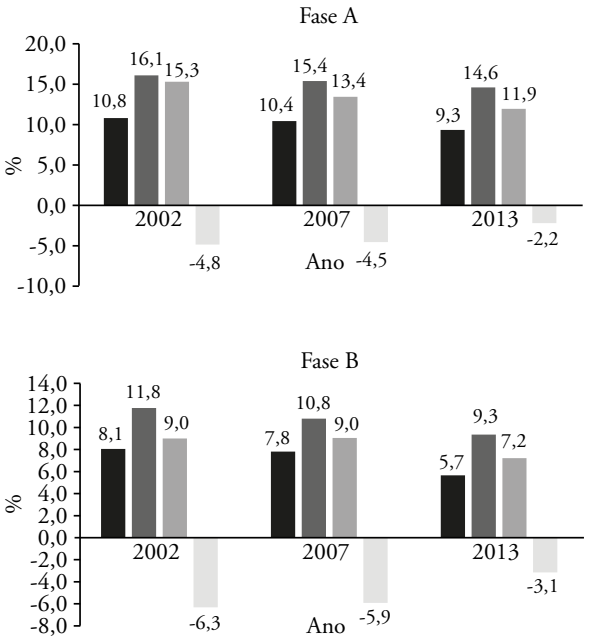

Escolaridade do indivíduo

Escolaridade requerida

Sobre-educação

Subeducaçāo

Fonte: $\operatorname{IBGE}(2002 b ; 2007 b ; 2013 b)$.

de 15 anos ou mais de idade, para os anos de 2002, 2007 e 2013. Para ambos os modelos, as análises foram feitas em duas fases: na fase A, avaliamos apenas o efeito da escolaridade de cada um dos modelos; na fase B, inserimos as variáveis de controle, que dizem respeito aos aspectos discriminadores do mercado de trabalho para verificar o comportamento da escolaridade quando controlada por essas variáveis ${ }^{5}$. Nossa pretensão foi contrapor o efeito da escolaridade nos dois modelos analíticos para cada um dos contextos e conjunturas do mercado de trabalho.

Podemos constatar que o efeito da escolaridade dos indivíduos é sempre menor do que o efeito da escolaridade requerida pela ocupação para as regióes metropolitanas do Rio de Janeiro e de São Paulo, em todos os anos analisados. Isso significa que mais importante do que a escolaridade dos indivíduos é a escolaridade exigida pela ocupação. Em 2002, por exemplo, o efeito da escolaridade dos indivíduos (fase A), na regiáo metropolitana do 
Rio de Janeiro era de $11,6 \%$, enquanto o efeito da escolaridade requerida pela ocupaçáo (fase A) era de 16,2\%. Significa que cada ano a mais de escolaridade dos indivíduos, seu rendimento aumenta, em média, em 11,6\%, ao passo que cada ano a mais de escolaridade requerida pela ocupação aumenta o rendimento dos indivíduos, em média, em 16,2\%. Situação semelhante também é observada para a regiáo metropolitana de São Paulo no mesmo ano, sendo que o efeito da escolaridade dos indivíduos era de $10,8 \%$, enquanto que a escolaridade requerida era de $16,1 \%$.

Quando observamos ao longo do tempo, constatamos que o efeito da escolaridade requerida pela ocupaçáo é sempre maior do que o efeito da escolaridade dos indivíduos. Porém, constatamos também que em ambos os modelos, e nas duas regióes metropolitanas, há redução do coeficiente que mensura o efeito $\mathrm{da}$ escolaridade sobre os diferenciais de rendimento. $\mathrm{Na}$ medida em que no decurso do tempo o mercado de trabalho se tornou mais expansivo, dada a redução do desemprego, a escolaridade tendeu a perder força como mecanismo explicativo para os diferenciais de renda. Isso porque, numa situação de expansão do mercado de trabalho, torna-se mais difícil discriminar por meio da escolaridade as oportunidades de ocupação que apesentam melhores rendimentos, apesar da escolaridade ainda se manter como um atributo importante. Nesse caso, o mecanismo da fila proposto por Thurow (1972) tende a se "enfraquecer", pois, num contexto de grande demanda por trabalho, a escolaridade deixa de servir como instrumento de discriminação da oferta de trabalho existente no mercado. Na verdade, essa interpretação pode ser feita no sentido de que a competiçáo por emprego tende a ser menor, dada a existência de grande demanda por trabalho. Por sua vez, isso provoca "enfraquecimento" da escolaridade como mecanismo de seleçáo entre os indivíduos no mercado de trabalho.

Podemos verificar que o efeito da sobre-educação sobre os diferenciais de renda é sempre positivo e menor do que o efeito da ocupação requerida, de acordo com os fatos estilizados observados nas pesquisas especializadas. Porém, como apontado por Hartog (2000), nos países desenvolvidos esse efeito corresponde entre a metade e $75 \%$ do efeito da escolaridade requerida. Nas duas regióes metropolitanas, o efeito da sobre-educação é superior a 90\% em 2002, mas apresenta redução em 2007 e 2013, chegando nesse último ano a pouco mais de 80\%, o que demonstra que com o aumento da situação de sobre-educação no mercado de trabalho dessas duas metrópoles, o seu efeito tende a ser semelhante ao observado nos países economicamente desenvolvidos.

O resultado encontrado para a subeducação também corresponde aos fatos estilizados das pesquisas especializadas. Isso porque o efeito da subeducação é sempre negativo e inferior (em módulos) em relação ao efeito da sobre-educação. Significa que a penalidade pela subeducação é inferior ao prêmio obtido no mercado de trabalho pela sobre-educaçáo. Também se constata 
que o efeito da subeducação apresenta redução ao longo do período analisado, o que pode decorrer, nesse caso, de redução da proporção de pessoas que se encontram nessa situação.

Quando se acrescentam as variáveis de controle (fase B), há redução do efeito da escolaridade, em ambos os modelos e nas duas metrópoles. Isso denota que parte da explicação da escolaridade decorre dos aspectos discriminadores do mercado de trabalho, como são o sexo, a cor ou raça, a posição social e a localização residencial na metrópole, além da experiência do trabalho.

Mesmo com a incorporação das variáveis de controle, observamos comportamento semelhante na comparação entre a escolaridade do indivíduo e a escolaridade requerida pela ocupação. Isto é, o retorno monetário decorrente da escolaridade requerida pela ocupação é sempre maior do que o retorno monetário da escolaridade do indivíduo, apesar de em ambas as situaçóes a escolaridade apresentar retorno monetário inferior decorrente do acréscimo das variáveis de controle. Além disso, podemos observar que a situação de sobre-educação apresenta coeficiente positivo e sempre menor do que a escolaridade requerida. Já a situação de subeducação apresenta coeficiente negativo e é menor (em módulo) do que o retorno da sobre-educação.

Para evidenciar os retornos monetários decorrente do modelo ORU, realizamos o cálculo dos valores preditos para indivíduos que apresentam a mesma característica pessoal, possuem a mesma posição social e residem em iguais condiçóes na metrópole. Ou seja, consideramos indivíduos homens, de cor branca, experiência de trabalho compatível com a média dos indivíduos, cuja posição social corresponde às ocupaçôes médias e que moram no núcleo metropolitano. A única diferença entre esses indivíduos é que um deles possui 11 anos de escolaridade e encontra-se em ocupação cuja exigência de escolaridade é também de 11 anos; outro indivíduo, cuja escolaridade é de 15 anos, porém se encontra em uma ocupação que exige 11 anos de escolaridade; e um terceiro indivíduo, cuja escolaridade é de 15 anos, e se encontra em uma ocupação que exige 15 anos de escolaridade. Realizamos essa comparaçáo para os anos de 2002 e 2013 para as regiốes metropolitanas do Rio de Janeiro e de Sáo Paulo. Os resultados estão expostos, respectivamente, nos Gráficos 4 e 5.

O indivíduo que apresenta situação de sobre-educação ganha, em média, mais do que o indivíduo que possui o mesmo nível de escolaridade, desde que esteja em uma ocupação compatível com sua escolaridade. Isso significa que há um prêmio para os indivíduos que apresentam maior escolaridade em relação ao que é exigido pela ocupação. Porém, esse indivíduo que possui 15 anos de escolaridade em uma ocupação que exige 11 anos de escolaridade ganha, em média, menos do que o indivíduo que dispóe da mesma escolaridade, mas que se encontra numa ocupação em que há compatibilidade entre a sua escolaridade e 


\section{Gráfico 4}

Valores preditos dos diferenciais de rendimento do trabalho principal de pessoas ocupadas de 15 anos e mais de idade, regiāo metropolitana do Rio de Janeiro, 2002 e 2013.

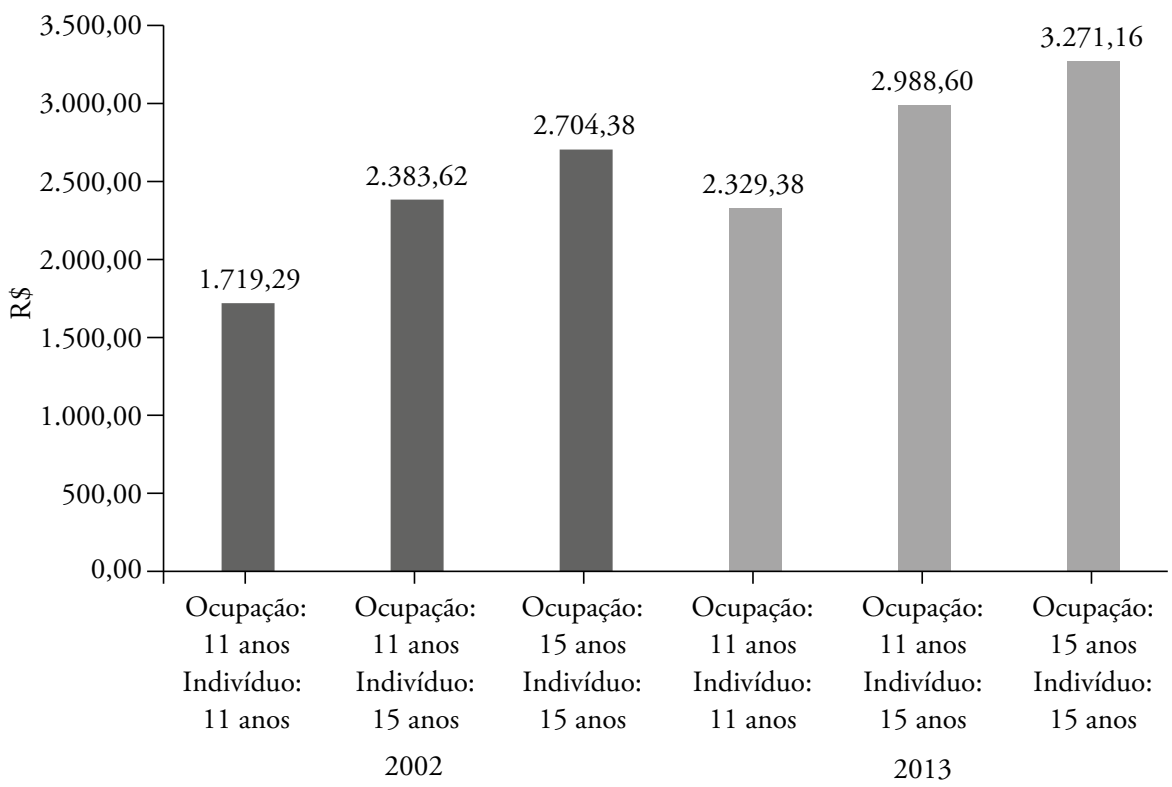

Fonte: Pesquisa Nacional por Amostra de Domicílios (IBGE, 2002b, 2013b).

Nota: Valores corrigido pelo Índice Nacional de Preços ao Consumidor, de setembro de 2014.

a escolaridade exigida pela ocupação. Ou seja, o prêmio obtido pela situação de sobre-educação não compensa o retorno monetário se os indivíduos estiverem em ocupaçôes compatíveis com a sua escolaridade. Esse comportamento é observado nas duas regiōes metropolitanas na comparação entre os anos de 2002 e 2013 mesmo com o aumento do nível de renda ocorrido nesse período, como podemos observar pelos valores apresentados nos gráficos ilustrativos.

\section{Considerações finais}

O objetivo deste trabalho foi confrontar dois modelos analíticos utilizados para analisar o efeito da escolaridade sobre os diferenciais de rendimentos obtidos pelos indivíduos no mercado de trabalho (Mincer e ORU) para períodos diferentes da conjuntura econômica brasileira (2002, 2007 e 2013) nas duas principais metrópoles do país: a região metropolitana de São Paulo e a região metropolitana do Rio de Janeiro. Observamos, para esses contextos territoriais, 


\section{Gráfico 5}

Valores preditos dos diferenciais de rendimento do trabalho principal de pessoas ocupadas de 15 anos e mais de idade, regiáo metropolitana de São Paulo, 2002 e 2013.

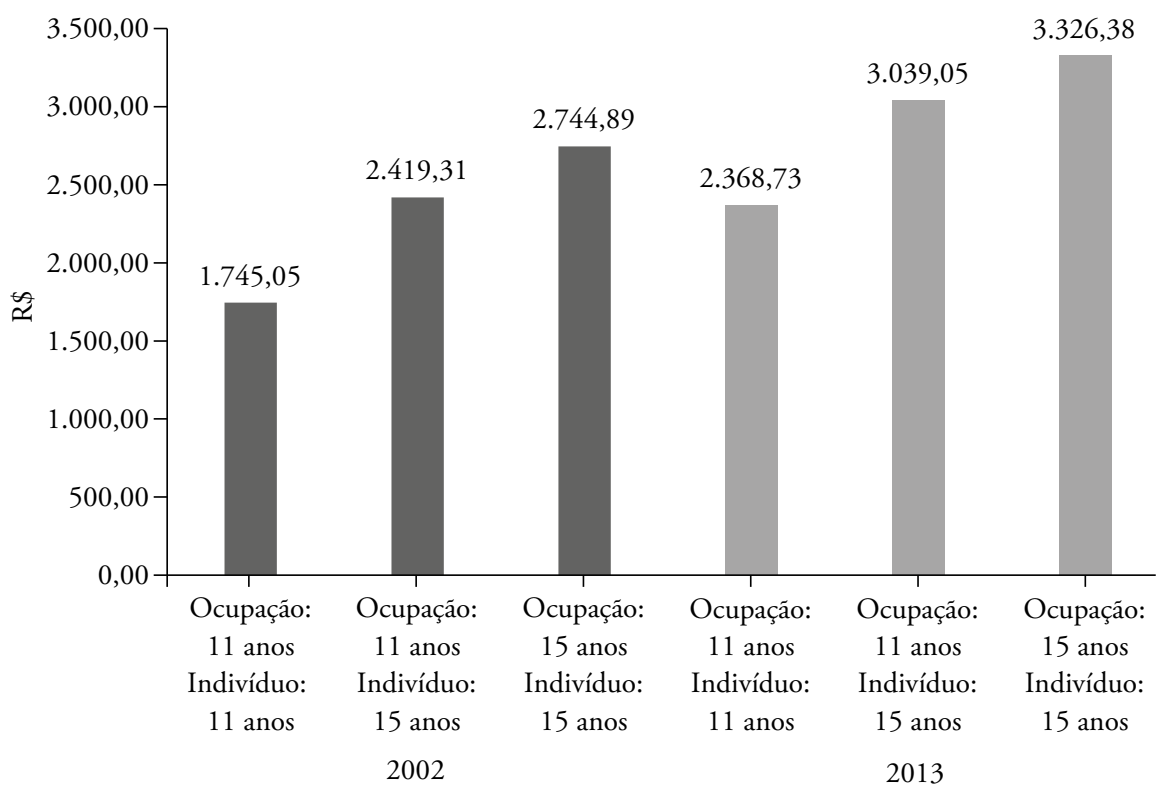

Fonte: Pesquisa Nacional por Amostra de Domicílios (IBGE, 2002b, 2013b).

Nota: Valores corrigido pelo Índice Nacional de Preços ao Consumidor, de setembro de 2014.

que os resultados apresentados foram compatíveis com o observado nos países desenvolvidos, à proporçâo que o retorno monetário decorrente da escolaridade requerida se mostrou mais elevado do que o retorno da escolaridade dos indivíduos. O retorno monetário derivado da sobre-educação foi positivo, mas inferior ao retorno decorrente da escolaridade requerida - nesse caso, mais elevado do que o observado nos países desenvolvidos, apesar de alcançar relativa aproximaçáo. O retorno decorrente da subeducação foi negativo e inferior àquele decorrente da sobre-educação, demonstrando que a penalidade para situação de subeducação é menor do que o prêmio para situação de sobre-educação.

Vimos que, de modo geral, a escolaridade reduz seu efeito ao longo do tempo como explicação dos retornos monetários. Isso pode se dever tanto à mudança conjuntural do mercado de trabalho - na medida em que houve redução da taxa de desemprego, o que diminui a competiçâo por emprego entre os indivíduos e, portanto, a escolaridade se "enfraquece" como atributo de diferenciação no mercado de trabalho - , quanto ao aumento do nível de escolaridade da população economicamente ativa, como temos observado nos últimos anos no país. Ou pode decorrer, 
principalmente, do efeito conjugado desses dois fatores de modo que, num contexto expansionista do mercado de trabalho, associado ao aumento da escolaridade da populaçáo, torna-se cada vez mais importante a avaliaçáo da sobre-educaçáo como retorno monetário no mercado de trabalho. Isso porque numa conjuntura em que haja excessiva oferta de mão de obra escolarizada, aqueles que apresentam os maiores níveis de escolaridade utilizarão esses atributos para conseguir melhor inserção no mercado de trabalho no que se refere à obtenção de renda.

$\mathrm{O}$ que podemos observar foi que indivíduos em iguais características pessoais, na mesma posição social e moradores do mesmo lugar na metrópole apresentam diferenças em termos de obtenção de renda, dependendo da sua situação em possuir ou não sobre-educação. $\mathrm{O}$ exemplo ilustrado na seção anterior foi capaz de demonstrar que indivíduos com maior escolaridade do que a requerida pela ocupação ganham mais do que indivíduos que estão em condiçôes compatíveis referente à escolaridade exigida pela ocupação e à sua escolaridade apresentada; porém, ganham menos do que indivíduos que estão em situação compatível de escolaridade, mas num nível mais elevado. Isso significa que o prêmio pela sobre-educação não é capaz de compensar o retorno monetário para os indivíduos, caso eles se inserissem em ocupaçôes cuja exigência de escolaridade fosse compatível com a deles.

Posto que o Brasil tem apresentado, cada vez mais, aumento do nível de escolaridade de sua população, configurando de modo crescente, como vimos, pessoas em situação de sobre-educação no mercado de trabalho, torna-se importante avançar na análise dessas situaçóes para melhor compreensão das diferenças de obtenção de renda entre os indivíduos no mercado de trabalho. Nesse sentido, é preciso compreender o porquê da escolaridade requerida pela ocupação ser mais importante como explicação para os diferenciais de renda entre os indivíduos do que a escolaridade por eles apresentada, ao mesmo tempo em que a escolaridade dos indivíduos serve também para diferenciá-los em termos dos retornos monetários apresentados. Assim, procuramos com este trabalho servir de estímulo para o aprofundamento de tais questóes, tanto empírica quanto teoricamente.

\section{Notas}

1. O modelo de Mincer considera como variável dependente o logaritmo natural do rendimento por hora e como variáveis explicativas os anos de escolaridade dos indivíduos e variável proxy de experiência de trabalho.

2. Apesar do modelo analítico proposto ser denominado pela sigla em inglês ORU (overeducation, required and undereducation), ao longo do trabalho vamos utilizar o correspondente em português. Assim overeducation será mencionada como sobre-educação e undereducation será mencionada como subeducação.

3. Até recentemente, o ensino fundamental correspondia ao total de 8 anos de escolaridade, assim como o ensino médio correspondia a 11 anos de escolaridade, e o ensino superior, em geral, 
correspondia a 15 anos de escolaridade. Não é à toa que são nessas faixas em que há maior proporção de pessoas na distribuiçáo da população ocupada segundo os anos de escolaridade, porque elas demonstram mudança de fase de escolaridade.

4. Ao invés de utilizar o peso original da PNAD, calculamos o peso amostral do seguinte modo:

$\mathrm{PA}=(\mathrm{Isp} / \mathrm{Icp}) \times \mathrm{PO}$

Onde:

$\mathrm{PA}=$ peso amostral;

$\mathrm{PO}=$ peso original;

Isp = número de indivíduos (casos) sem peso;

Icp = número de indivíduos (casos) com peso.

5. Por falta de espaço, evitamos tecer consideraçôes sobre o efeito dessas variáveis de controle sobre os diferenciais de renda entre os indivíduos no mercado de trabalho.

\section{Referências}

BECKER, G.S. Human capital: a theoretical and empirical analysis, with special reference to education. Massachusetts: National Bureau of Economic Research, 1964.

DIAZ, M.D.M.; MACHADO, L. Overeducation e undereducation no Brasil: incidência e retornos. Estudos Econômicos, v. 38, n. 3, p. 431-460, jul./set. 2008.

DUNCAN, G.J.; HOFFMAN, S.D. The incidence and wage effects of overeducation. Economics of Education Review, v. 1, n. 1, p. 75-86, 1981.

ESTEVES, L.A. Incompatibilidade escolaridade-ocupação e salários: evidências de uma empresa industrial brasileira. Revista Brasileira de Economia, v. 63 n. 2, p. 77-90, abr./jun. 2009.

GROSSBARD, S. (Ed.). Jacob Mincer: a pioneer of modern labor economics. New York: Springer, 2006. $210 \mathrm{p}$.

HARTOG, J. Over-education and earnings: where are we, where should we go? Economics of Education Review, n. 19, n. 2, p. 131-147, abr. 2000.

IBGE - INSTITUTO BRASILEIRO DE GEOGRAFIA E ESTATÍSTICA. Pesquisa Mensal de Emprego. Rio de Janeiro: IBGE, 2002a.

. Pesquisa Nacional por Amostra de Domicílio. Rio de Janeiro: IBGE, $2002 \mathrm{~b}$.

. Pesquisa Mensal de Emprego. Rio de Janeiro: IBGE, 2007a.

. Pesquisa Nacional por Amostra de Domicílio. Rio de Janeiro: IBGE, $2007 \mathrm{~b}$.

. Pesquisa Mensal de Emprego. Rio de Janeiro: IBGE, 2013 a.

Pesquisa Nacional por Amostra de Domicílio. Rio de Janeiro: IBGE, 2013 b.

MACHADO, A.F.; OLIVEIRA, A.M.H.C.; CARVALHO, N.F. Tipologia de qualificaçâo da força de trabalho: uma proposta a partir da noção de incompatibilidade entre ocupação e escolaridade. Texto para discussão n. 218. Belo Horizonte: UFMG/Cedeplar, 2003. 
MCGUINNESS, S. Overeducation in the Labour Market. Journal of Economic Surveys, v. 20, n. 3, p. 387-418, 2006.

MINCER, J.A. Schooling, experience, and earnings. New York: Columbia University Press, 1974. $152 \mathrm{p}$.

. Investment in human capital and personal income distribution. Journal of Political Economy, v. 66, n. 4, p. 281-302, ago. 1958.

SANTOS, A. M. Overeducation no mercado de trabalho brasileiro. Revista Brasileira de Economia de Empresas, v. 2, n. 2, p. 1-22, 2002.

SCHULTZ, T.W. Investment in human capital. The American Economic Review, v. 51, n. 1 , p. $1-17$, mar. 1961 .

THUROW, L.C. Education and economic equality. The Public Interest, n. 28, p. 66-81, 1972.

VERDUGO, R.R.; VERDUGO, N.T. The impact of surplus schooling on earnings: some additional findings. The Journal of Human Resources, v. 24, n. 4, p. 629-643, 1989.

VIANNA, C.H.; OLIVEIRA, A.M.H.C. Sobre-escolarização nas ocupaçóes brasileiras: uma análise dos efeitos de idade, período e coorte. In: 38 ENCONTRO NACIONAL DE ECONOMIA, Salvador, BA. Salvador: ANPEC, 2010.

Recebido em 10 de setembro de 2015.

Aprovado em 31 de maio de 2016. 